\title{
The Effect of Circadian and Sleep Disruptions on Obesity Risk
}

\author{
Junghyun Noh* \\ Department of Internal Medicine, Inje University Ilsan Paik Hospital, Goyang, Korea
}

The prevalence of obesity continues to increase worldwide. Multiple risk factors and complex mechanisms are involved in the development and maintenance of obesity. Recently, emerging evidence has indicated that the circadian rhythm is important for regulating metabolism because the circadian system modulates energy metabolism and enhances certain energetic activities during day and night. Lifestyle also has a great impact on the circadian system. In addition to traditional risk factors, sleep and circadian disruptions are known modifiable risk factors for obesity and other metabolic disorders. This paper reviews recent evidence of relationships between energy metabolism and the circadian system and discusses how sleep disruption and circadian misalignment influence the development of obesity.

Key words: Obesity, Circadian rhythm, Sleep wake disorders

\author{
Received March 6, 2018 \\ Reviewed March 8, 2018 \\ Accepted March 15, 2018 \\ *Corresponding author \\ Junghyun Noh \\ (iD) \\ https://orcid.org/0000-0002-7964-0515 \\ Department of Internal Medicine, Inje \\ University Ilsan Paik Hospital, 170 \\ Juhwa-ro, Ilsanseo-gu, Goyang 10380, \\ Korea \\ Tel: $+82-31-910-7930$ \\ Fax: +82-31-910-7217 \\ E-mail:jhnoh@paik.ac.kr
}

\section{INTRODUCTION}

Obesity rates are increasing throughout the world, including Korea. ${ }^{1}$ The latest estimates in Korea suggest that approximately $37.6 \%$ of men and $48.5 \%$ of women have diabetes, and $35.7 \%$ of Korean men and $45.2 \%$ of Korean women without diabetes are obese. ${ }^{1}$ Obese people are at an increased risk for many comorbidities, including insulin resistance, type 2 diabetes mellitus (T2DM), high blood pressure, and cardiovascular disease, compared to those at a healthy weight. Genetics, an unhealthy diet, and inactivity are common causes of adverse metabolic health, including obesity and glucose intolerance. ${ }^{2}$ However, these well-known factors do not fully explain the significant epidemic of obesity. Increasing evidence from both laboratory and epidemiological studies indicates that sleep and circadian rhythm disturbances are novel risk factors for obesity and obesity-related comorbidities. ${ }^{3-5}$ Sleep is an important modulator of neuroendocrine function and glucose metabolism, and sleep loss has been shown to result in metabolic and endocrine alterations, including impaired glucose tolerance and modification of hormones that affect appetite regulation. These results suggest that improvement of sleep and circadian disruptions may promote metabolic health. This review is focused on the relationship between sleep and circadian disruption and obesity risk at the population and laboratory levels.

\section{CIRCADIAN CLOCK SYSTEM}

The circadian system consists of two parts: central and peripher- 
al clocks. The central circadian clock is located at the hypothalamic suprachiasmatic nucleus (SCN). The central circadian clock, which is entrained by light, controls diurnal rhythm and thereby synchronizes metabolic functions during the daytime. Diffusible factors, such as cortisol and melatonin, and synaptic projections are considered to be the main pathways of the central clock. ${ }^{6,7} \mathrm{~A}$ series of peripheral clocks is located in almost every cell throughout the body, including the liver, gastrointestinal tract, pancreas, skeletal muscle, and adipose tissue. ${ }^{8}$ Peripheral clocks receive signals from central clocks through hormones and synaptic projections, including the autonomic nervous system, and external factors, such as light, sleep, physical activity, and feeding. Peripheral clocks have their own autonomous rhythms consist of a transcriptional-translational feedback loop (TTFL) made up by a set of clock genes involving, circadian locomotor output cycles kaput (CLOCK) and brain and muscle aryl hydrocarbon receptor nuclear translocatorlike 1 (BMAL1) activators and their target genes named Period (Per1, Per2, Per3), cryptochrome (Cry1, Cry2) and Rev-erba, whose gene products form a negative feedback repressor complex (Fig. 1). ${ }^{9}$ The TTFL operates on a 24 -hour cycle. The individual cellular clocks have to be synchronized to produce a physiologic circadian clock system. A disturbance of any stage in this mechanism initiates misalignment of the circadian clock system and induces multiple metabolic effects.

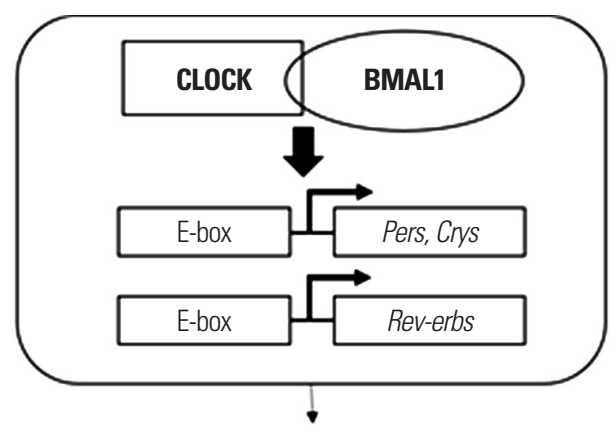

Clock-controlled genes

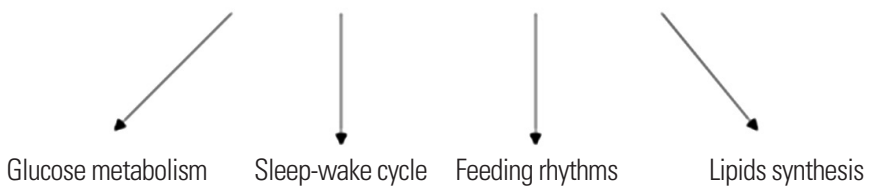

Figure 1. A schematic diagram of the regulatory role of the cellular circadian CLOCK system in the metabolic pathway. CLOCK, circadian locomotor output cycles kaput; BMAL1, brain and muscle aryl hydrocarbon receptor nuclear translocator-like 1

\section{CIRCADIAN SYSTEM AND ENERGY METABOLISM}

There is clear evidence that the circadian system and energy metabolism are intimately conjugated at both the central and peripheral levels. In studies with single-celled cyanobacteria or red blood cells, the production of adenosine triphosphate (ATP) induces a clear 24-hour circadian rhythm. In cyanobacteria, ATP production by the oxidative phosphorylation of adenosine diphosphate (ADP) follows a circadian rhythm that is synchronized to a 24-hour lightdark cycle. ${ }^{10}$ When cyanobacteria are subjected to an abnormal light-dark cycle with an 8-hour dark period in the daytime, which causes a circadian misalignment, the ATP to ADP ratio and enzymatic reactions for ATP metabolism are reduced..$^{10}$ In an animal study, transcript levels of Niemann-Pick disease, type $\mathrm{Cl}$ and other enzymes involved in cholesterol synthesis and degradation in lever exhibited a circadian rhythm. ${ }^{11}$ This result supports that the gene expressions coding for metabolically important enzymes are regulated by a circadian clock mechanism.

Direct regulation of the circadian system on energy metabolism occurs via binding of CLOCK and BMAL1 to E-box elements in the promoters of a gene. ${ }^{12,13}$ Mice with a mutation of the CLOCK gene not only show an altered and blunted circadian rhythm for wheel running in comparison with heterozygous mice but also exhibit a decrease in energy expenditure along with an increase in caloric intake and body mass. ${ }^{14}$ Dysregulation of BMAL1 in mice leads to changes in energy and lipid homeostasis similar to those of the metabolic syndrome. BMAL1 knockout (KO) mice exhibited suppression of the diurnal variation in glucose and triglyceride levels. ${ }^{15}$ Furthermore, Rev-erb KO mice showed circadian arrhythmicity in wheel-running activity. ${ }^{16}$ Several transcription factors regulated by the CLOCK system are also known to control metabolic genes, including the $\mathrm{D}$ site of albumin promoter binding protein (DBP), thyrotroph embryonic factor (TEF), hepatic leukemia factor (HLF), and nuclear receptors. ${ }^{17}$ Peroxisome proliferator-activated receptor gamma coactivator1- $\alpha$, a nuclear receptor involved in lipid metabolism, has been suggested to be the key integrator of CLOCK. ${ }^{18}$ Its activity is impaired in mice lacking the three circadian proline and acidic amino acid-rich basic leucine zipper factors DBP, TEF, and HLF. ${ }^{19}$ Less is known about the relationship be- 
tween energy metabolism and the circadian system in humans. Cross-sectional studies in humans have reported associations between the CLOCK locus and the prevalence of obesity, plasma glucose levels hypertension and $\mathrm{T}_{2} \mathrm{DM}^{20}$, which suggests the integration between energy metabolism and the circadian system.

\section{DISRUPTED SLEEP AND OBESITY}

Insufficient and disrupted sleep is common in modern society. The American Academy of Sleep Medicine and Sleep Research Society recommends that 7 or more hours of sleep are necessary each day to maintain optimal health and functioning. ${ }^{21}$ However, it is unclear that more than 9 hours of sleep or less than 7 hours are inappropriate. A short sleep period has been demonstrated to have a relationship with obesity in numerous studies. A meta-analysis of 36 cross-sectional studies showed that a short sleep duration was independently associated with weight gain, particularly in younger age groups. ${ }^{22}$ Theoretically, the increased energy expenditure that results from longer periods of wakefulness should cause weight loss, but short sleep generally produces weight gain instead. It has been suggested that the paradoxical relationship between short sleep and obesity in humans is mediated by increased dietary intake and altered physical activity. The increase in food intake that resulted when sleep was restricted in healthy men in several studies might induce weight gain. ${ }^{23-25}$ Epidemiologic studies that have examined sleep duration in relation to diet have indicated that a higher fat intake, lower quality diets and irregular eating behavior occur during sleep restriction. ${ }^{26}$ Disrupted sleep is now regarded as a new and important risk factor for abnormal lifestyle behavior, especially unhealthy eating.

The mechanism of increased food intake during sleep disruption may be varied. Sleep restriction induces changes in appetitive hormones, such as ghrelin, as well as increased hunger and appetite in human studies. Short sleepers generally have reduced leptin, elevated ghrelin and increased weight compared to average sleepers. ${ }^{27} \mathrm{~A}$ functional magnetic resonance imaging study also identified increased activation of brain regions that may be sensitive to food stimulation during sleep restriction. ${ }^{28,29}$ It has also been suggested that duration is not the only factor that plays an important role in obesity; sleep quality has been implicated as well. In a study that in- cluded 522 patients with newly diagnosed T2DM, the average number of night awakenings was positively correlated with body mass index (BMI). ${ }^{30}$ In a systemic review of 16 articles, six CLOCK single nucleotide polymorphisms were associated with sleep duration, and three variants were correlated with energy intake variables. ${ }^{31}$ These genetic variants may assist in identifying individuals more prone to overeating and gaining weight when experiencing sleep deprivation.

\section{CIRCADIAN MISALIGNMENT AND OBESITY}

As previously described, the circadian system and energy metabolism are tightly integrated with and synchronized to the environment. Circadian misalignment commonly occurs in shift workers and is defined as inappropriately timed sleep and wake cycles or the misalignment of sleep and wake with eating rhythms. Nightshift workers who suffer from circadian misalignment have a higher risk of obesity ${ }^{32,33}$, cardiovascular disease and diabetes. ${ }^{34}$ In a systemic review and meta-analysis of 28 studies, the overall odds ratio of night-shift workers was 1.23 (95\% confidence interval, 1.171.29) for the risk of obesity and overweight. Shift workers were also at higher risk of abdominal obesity than other obesity types (odds ratio, 1.35). ${ }^{35}$ Light at night (LAN) is a major precipitating factor of a disrupted circadian system. The retina senses light, and retinal ganglion cells project to the neurons of the $\mathrm{SCN}$, evoking a signal that leads to changes in Per 1 and Per 2 CLOCK gene expression. As a consequence, a shift in the phase of the circadian oscillator in the SCN results in a shift of the circadian system in the body. Several studies have found that shift workers have a higher prevalence of obesity and support that artificial lighting may contribute to the increased prevalence of metabolic disorders. ${ }^{36,37}$ Exposing mice to constant bright light throughout a 24-hour period resulted in an increased body mass by shifting the time of food intake despite a lack of increased caloric intake and no variation in activity level..$^{38}$ In humans, increased levels of LAN exposure were associated with an increased obesity rate in 100,000 women. ${ }^{39}$ Unusual meal timing is also an important factor in the development of obesity associated with circadian disruption. In rodent models, eating only during habitual sleep time increases body mass compared 
with eating during habitual wake time even when the caloric intake and locomotor activity were similar. ${ }^{40}$ A recent study uncovered an endogenous circadian rhythm for hunger; hunger senses are highest in the biological evening at about $8 \mathrm{PM}$ and lowest around 8 AM. This observation supports a limited caloric intake after a long overnight fast. ${ }^{41}$ However, when food is easily accessible overnight, eating at night is associated with unwanted weight gain. Another study also showed that late sleepers consumed more calories at dinner and after 8:00 PM, had a higher fast food and full-calorie soda intake and lower fruit and vegetable consumption and had a higher BMI. ${ }^{42}$ Night eating syndrome, diagnosed as (1) no appetite for breakfast, (2) consumption of $\geq 50 \%$ of daily energy after 7:00 $\mathrm{PM}$, and (3) sleep difficulties $\geq 3$ night/wk, is associated with a higher BMI and binge eating tendencies. ${ }^{43}$ The decreased total energy expenditure in response to eating meals at inappropriate times may contribute to the mechanism of unwanted weight gain and obesity associated with circadian misalignment. In a study of 14 adults in a 6-day inpatient simulated shiftwork protocol, sleep duration during the biological day and wakefulness and eating during the biological night in a shift-work model decreased the participants' 24-hour energy expenditure. ${ }^{44}$

\section{CONCLUSION}

There is strong evidence that disrupted sleep and circadian misalignment contribute to obesity and metabolic disorders. A short duration and poor quality of sleep are considered risk factors for the development of obesity. The inappropriate circadian timing of food intake is also regarded as a contributing factor for obesity. Greater weight gain and an altered glucose and energy metabolism occur when food intake during the biological night is increased in humans. Additional research will be needed to understand the mechanisms by which sleep and circadian disruption contribute to metabolic dysregulation and also to determine the potential for improving sleep and circadian rhythms to promote metabolic health.

\section{CONFLICTS OF INTEREST}

The author declares no conflict of interest.

\section{REFERENCES}

1. Noh J, Han KD, Ko SH, Ko KS, Park CY. Trends in the pervasiveness of type 2 diabetes, impaired fasting glucose and comorbidities during an 8-year-follow-up of nationwide Korean population. Sci Rep 2017;7:46656.

2. Stenvinkel P. Obesity: a disease with many aetiologies disguised in the same oversized phenotype. Has the overeating theory failed? Nephrol Dial Transplant 2015;30:1656-64.

3. Markwald RR, Wright KP Jr. Circadian misalignment and sleep disruption in shift work: implications for fatigue and risk of weight gain and obesity. In: Shiromani P, Horvath T, Redline S, Van Cauter E, editors. Sleep loss and obesity. New York: Springer; 2012. p. 101-18.

4. Depner CM, Stothard ER, Wright KP Jr. Metabolic consequences of sleep and circadian disorders. Curr Diab Rep 2014; 14:507.

5. Arble DM, Bass J, Behn CD, Butler MP, Challet E, Czeisler C, et al. Impact of sleep and circadian disruption on energy balance and diabetes: a summary of workshop discussions. Sleep 2015;38:1849-60.

6. Mohawk JA, Green CB, Takahashi JS. Central and peripheral circadian clocks in mammals. Annu Rev Neurosci 2012;35: 445-62.

7. Buijs RM, Kalsbeek A. Hypothalamic integration of central and peripheral clocks. Nat Rev Neurosci 2001;2:521-6.

8. Dibner C, Schibler U, Albrecht U. The mammalian circadian timing system: organization and coordination of central and peripheral clocks. Annu Rev Physiol 2010;72:517-49.

9. Partch CL, Green CB, Takahashi JS. Molecular architecture of the mammalian circadian clock. Trends Cell Biol 2014;24:909.

10. Rust MJ, Golden SS, O’Shea EK. Light-driven changes in energy metabolism directly entrain the cyanobacterial circadian oscillator. Science 2011;331:220-3.

11. Panda S, Antoch MP, Miller BH, Su AI, Schook AB, Straume $\mathrm{M}$, et al. Coordinated transcription of key pathways in the mouse by the circadian clock. Cell 2002;109:307-20.

12. Nakahata Y, Sahar S, Astarita G, Kaluzova M, Sassone-Corsi P. Circadian control of the NAD+ salvage pathway by CLOCK- 
SIRT1. Science 2009;324:654-7.

13. Asher G, Gatfield D, Stratmann M, Reinke H, Dibner C, Kreppel F, et al. SIRT1 regulates circadian clock gene expression through PER2 deacetylation. Cell 2008;134:317-28.

14. Turek FW, Joshu C, Kohsaka A, Lin E, Ivanova G, McDearmon E, et al. Obesity and metabolic syndrome in circadian Clock mutant mice. Science 2005;308:1043-5.

15. Shimba S, Ogawa T, Hitosugi S, Ichihashi Y, Nakadaira Y, Kobayashi $\mathrm{M}$, et al. Deficient of a clock gene, brain and muscle Arnt-like protein-1 (BMAL1), induces dyslipidemia and ectopic fat formation. PLoS One 2011;6:e25231.

16. Cho H, Zhao X, Hatori M, Yu RT, Barish GD, Lam MT, et al. Regulation of circadian behaviour and metabolism by REVERB- $\alpha$ and REV-ERB- $\beta$. Nature 2012;485:123-7.

17. Gachon F, Olela FF, Schaad O, Descombes P, Schibler U. The circadian PAR-domain basic leucine zipper transcription factors DBP, TEF, and HLF modulate basal and inducible xenobiotic detoxification. Cell Metab 2006;4:25-36.

18. Lin JD, Liu C, Li S. Integration of energy metabolism and the mammalian clock. Cell Cycle 2008;7:453-7.

19. Gachon F, Leuenberger N, Claudel T, Gos P, Jouffe C, Fleury Olela F, et al. Proline- and acidic amino acid-rich basic leucine zipper proteins modulate peroxisome proliferator-activated receptor alpha (PPARalpha) activity. Proc Natl Acad Sci U S A 2011;108:4794-9.

20. Corella D, Asensio EM, Coltell O, Sorlí JV, Estruch R, Martínez-González MÁ, et al. CLOCK gene variation is associated with incidence of type- 2 diabetes and cardiovascular diseases in type-2 diabetic subjects: dietary modulation in the PREDIMED randomized trial. Cardiovasc Diabetol 2016;15:4.

21. Watson NF, Badr MS, Belenky G, Bliwise DL, Buxton OM, Buysse D, et al. Recommended amount of sleep for a healthy adult: a joint consensus statement of the American Academy of Sleep Medicine and Sleep Research Society. Sleep 2015;38: 843-4.

22. Patel SR, Hu FB. Short sleep duration and weight gain: a systematic review. Obesity (Silver Spring) 2008;16:643-53.

23. Markwald RR, Melanson EL, Smith MR, Higgins J, Perreault L, Eckel RH, et al. Impact of insufficient sleep on total daily energy expenditure, food intake, and weight gain. Proc Natl
Acad Sci U S A 2013;110:5695-700.

24. Brondel L, Romer MA, Nougues PM, Touyarou P, Davenne D. Acute partial sleep deprivation increases food intake in healthy men. Am J Clin Nutr 2010;91:1550-9.

25. Spaeth AM, Dinges DF, Goel N. Effects of experimental sleep restriction on weight gain, caloric intake, and meal timing in healthy adults. Sleep 2013;36:981-90.

26. Dashti HS, Scheer FA, Jacques PF, Lamon-Fava S, Ordovás JM. Short sleep duration and dietary intake: epidemiologic evidence, mechanisms, and health implications. Adv Nutr 2015;6:648-59.

27. Taheri S, Lin L, Austin D, Young T, Mignot E. Short sleep duration is associated with reduced leptin, elevated ghrelin, and increased body mass index. PLoS Med 2004; 1:e62.

28. St-Onge MP, McReynolds A, Trivedi ZB, Roberts AL, Sy M, Hirsch J. Sleep restriction leads to increased activation of brain regions sensitive to food stimuli. Am J Clin Nutr 2012;95:81824.

29. Greer SM, Goldstein AN, Walker MP. The impact of sleep deprivation on food desire in the human brain. Nat Commun 2013;4:2259.

30. Arora T, Chen MZ, Omar OM, Cooper AR, Andrews RC, Taheri S. An investigation of the associations among sleep duration and quality, body mass index and insulin resistance in newly diagnosed type 2 diabetes mellitus patients. Ther Adv Endocrinol Metab 2016;7:3-11.

31. Valladares M, Obregón AM, Chaput JP. Association between genetic variants of the clock gene and obesity and sleep duration. J Physiol Biochem 2015;71:855-60.

32. Di Lorenzo L, De Pergola G, Zocchetti C, L’Abbate N, Basso A, Pannacciulli N, et al. Effect of shift work on body mass index: results of a study performed in 319 glucose-tolerant men working in a Southern Italian industry. Int J Obes Relat Metab Disord 2003;27:1353-8.

33. Antunes LC, Levandovski R, Dantas G, Caumo W, Hidalgo MP. Obesity and shift work: chronobiological aspects. Nutr Res Rev 2010;23:155-68.

34. Vetter C, Devore EE, Wegrzyn LR, Massa J, Speizer FE, Kawachi I, et al. Association between rotating night shift work and risk of coronary heart disease among women. JAMA 
$2016 ; 315: 1726-34$.

35. Sun M, Feng W, Wang F, Li P, Li Z, Li M, et al. Meta-analysis on shift work and risks of specific obesity types. Obes Rev 2018;19:28-40.

36. Leproult R, Deliens G, Gilson M, Peigneux P. Beneficial impact of sleep extension on fasting insulin sensitivity in adults with habitual sleep restriction. Sleep 2015;38:707-15.

37. Killick R, Hoyos CM, Melehan KL, Dungan GC 2nd, Poh J, Liu PY. Metabolic and hormonal effects of 'catch-up' sleep in men with chronic, repetitive, lifestyle-driven sleep restriction. Clin Endocrinol (Oxf) 2015;83:498-507.

38. Fonken LK, Workman JL, Walton JC, Weil ZM, Morris JS, Haim A, et al. Light at night increases body mass by shifting the time of food intake. Proc Natl Acad Sci U S A 2010;107: 18664-9.

39. McFadden E, Jones ME, Schoemaker MJ, Ashworth A, Swerdlow AJ. The relationship between obesity and exposure to light at night: cross-sectional analyses of over 100,000 women in the Breakthrough Generations Study. Am J Epide- miol 2014;180:245-50.

40. Arble DM, Bass J, Laposky AD, Vitaterna MH, Turek FW. Circadian timing of food intake contributes to weight gain. Obesity (Silver Spring) 2009;17:2100-2.

41. Scheer FA, Morris CJ, Shea SA. The internal circadian clock increases hunger and appetite in the evening independent of food intake and other behaviors. Obesity (Silver Spring) 2013; 21:421-3.

42. Baron KG, Reid KJ, Kern AS, Zee PC. Role of sleep timing in caloric intake and BMI. Obesity (Silver Spring) 2011;19:1374 81.

43. Colles SL, Dixon JB, O’Brien PE. Night eating syndrome and nocturnal snacking: association with obesity, binge eating and psychological distress. Int J Obes (Lond) 2007;31:1722-30.

44. McHill AW, Melanson EL, Higgins J, Connick E, Moehlman TM, Stothard ER, et al. Impact of circadian misalignment on energy metabolism during simulated nightshift work. Proc Natl Acad Sci U S A 2014;111:17302-7. 